\title{
The Clinicopathological Features of Papillary Thyroid Carcinoma Patients with Positive Hepatitis B Surface Antigen
}

\author{
Zhenwei Zhong $^{\mathrm{a}, \mathrm{b}} \quad$ Jiru Yuan ${ }^{\mathrm{a}, \mathrm{b}} \quad$ Xiaohang Chen ${ }^{\mathrm{a}, \mathrm{b}} \quad$ Zhengbo Chen $^{\mathrm{a}}$ \\ Jialin $\mathrm{Du}^{\mathrm{a}}$ Zhijian Chen ${ }^{\mathrm{c}}$ Jian Kuang ${ }^{c}$ Shuzhen Cong ${ }^{\mathrm{d}}$ Jinghua Li ${ }^{\mathrm{e}}$ \\ Zeyu $\mathrm{Wu}^{\mathrm{a}}$ \\ aDepartment of General Surgery, Guangdong Provincial People's Hospital, Guangdong Academy of Medical \\ Sciences, Guangzhou, China; ${ }^{b}$ Shantou University Medical College, Shantou, China; ' $D$ Department of Endocrinology, \\ Guangdong Provincial People's Hospital, Guangdong Academy of Medical Sciences, Guangzhou, China; \\ ${ }^{\mathrm{d}}$ Department of Ultrasound, Guangdong Provincial People's Hospital, Guangdong Academy of Medical Sciences, \\ Guangzhou, China; 'Department of Pathological Medicine, Guangdong Provincial People's Hospital, Guangdong \\ Academy of Medical Sciences, Guangzhou, China
}

\section{Keywords}

Hepatitis B surface antigen - Central lymph node metastasis . Lymph node metastasis · Papillary thyroid carcinoma

\begin{abstract}
Background: Hepatitis B virus infection has been reported to be associated with some kinds of cancer. The aim of this study was to investigate clinicopathological features of papillary thyroid carcinoma (PTC) patients with positive hepatitis B surface antigen (HBsAg). Materials and Methods: A total of 569 PTC patients were analyzed retrospectively in this study. The relationships of $\mathrm{HBsAg}$ with clinicopathologic features of PTC were analyzed by univariate analysis. The relationships of central lymph node metastasis (CLNM) with clinicopathological features of PTC were analyzed by univariate and multivariate logistic regression analysis. Results: The incidence of CLNM in PTC with positive HBsAg was higher than that in PTC with negative HBsAg (71.4 vs. $60.0 \%, p=0.047$ ). Univariate analysis showed that positive $\mathrm{HBsAg}$ was significantly associated with bilateral tumors $(p=0.043)$ and lymph node metastasis (LNM) $(p=0.047)$ in PTC patients. In addition, the incidence of CLNM in patients with PTC was $61.7 \%$ (351 of 569 cases). Univariate analysis showed that CLNM of patients with PTC was significantly associated with positive
\end{abstract}

HBsAg ( $p=0.047)$. Multivariate logistic regression analysis revealed that positive HBsAg ( $p=0.038)$ was an independent predictor of CLNM in patients with PTC. Conclusions: The incidence of CLNM in PTC patients with positive HBsAg was significantly higher than that of patients with negative HBsAg. Positive HBsAg was correlated with LNM and bilateral tumors. In addition to gender, age $<45$ years, tumor size $>2$ $\mathrm{cm}$, and lateral LNM, positive HBsAg was also an independent predictor of CLNM in PTC patients.

(c) 2019 S. Karger AG, Basel

\section{Introduction}

The incidence of thyroid carcinoma, which is the most common endocrine malignancy, has increased rapidly [1, 2]. Papillary thyroid carcinoma (PTC) is the most common pathological subtype of thyroid carcinoma, accounting for over $80 \%$ of all thyroid malignancies [3, 4]. According to the classification of World Health Organization, papillary thyroid microcarcinoma (PTMC) is PTC with a maximum diameter $\leq 10 \mathrm{~mm}$ [5]. PTC with a max- 
imum diameter $>10 \mathrm{~mm}$ was defined as conventional PTC $[6,7]$. Chronic hepatitis B virus (HBV) infection is a global health problem. More than 2 billion people are estimated to be infected, and more than 350 million are chronic carriers of $\mathrm{HBV}[8,9]$. Variations of hepatitis B surface antigen (HBsAg) seroprevalence are wide - it is lowest in Norway and the UK (0.01\%) and highest (>20\%) in countries such as South Sudan and Kiribati [10, 11]. China was considered to have high prevalence $(>7 \%)$ of $\mathrm{HBV}$, as well as South East Asia, Africa, the Pacific Islands, and parts of the Middle East and Amazon [12, 13]. The USA, western Europe, Australia, and Japan were considered to have the lowest prevalence $(<2 \%)$ [8]. Therefore, HBV infection is still a significant socioeconomic problem in developing countries including China [14]. People with HBV infection are at increased risk of developing hepatic decompensation, cirrhosis, and hepatocellular carcinoma [9]. In addition, HBV infection has been associated with an increased risk of developing lots of malignancies such as stomach cancer, colorectal cancer, gallbladder and extrahepatic bile duct cancer, pancreatic cancer, lung cancer, kidney cancer, bladder cancer, uterine and corpus cancer, ovarian cancer, prostate cancer, breast cancer, skin cancer, non-Hodgkin's lymphoma, and leukemia [15-19]. However, there has been no study on the role of $\mathrm{HBV}$ infection in thyroid cancer. The purpose of this study was to investigate clinicopathological features of PTC patients with positive HBsAg.

\section{Materials and Methods}

In this study, we retrospectively analyzed a total of 569 PTC patients in the Department of General Surgery at Guangdong Provincial People's Hospital between March 2014 and September 2017. Preoperative assessment included ultrasonography, chest Xray, fluoro-18-deoxyglucose positron emission tomography (PET), computed tomography (CT) scan, and measurement of thyroglobulin, anti-thyroglobulin antibody, and thyroid stimulating hormone levels. In China, every preoperative patient was routinely examined for HBV serological markers to prevent cross-infection. HBV infection was diagnosed based on HBsAg positivity $[10,13]$. Ultrasonography was performed to assess the lymph node status and to confirm no lymph node involvement in all patients preoperatively. We used CT scan to examine suspicious invasion of the surrounding tissues or substernal thyroid cancers. PET scan was used in patients with suspected distant metastasis (such as lung or bone metastases). Patients with HBV infection with acute liver injury, previous neck, thyroid, or parathyroid surgery, history of neck radiation, or family history of cancers were excluded. The following information was collected from the medical records of the patients: gender, age, TNM staging, bilaterality, lymph node metastasis (LNM) status, tumor size, multifocality, capsular invasion, lymphovascular invasion, extrathyroidal invasion, recurrence stratification, and $\mathrm{HBsAg}$ status.

In this study, there were 390 (68.5\%) women and 179 (34.5\%) men. The mean age was $41.3 \pm 13.2$ years (range $5-82$ years). There were $222(39.0 \%)$ patients aged $\geq 45$ years and $347(61.0 \%)$ aged $<45$ years. We found $14.8 \%$ ( 84 of 569 cases) of patients had positive
HBsAg. The mean age of these 84 patients was $42.3 \pm 12.1$ years (range 18-74 years). All patients underwent bilateral central neck dissection (CND) in addition to total/near-total thyroidectomy. Ipsilateral therapeutic lateral lymph node dissection was performed in $122(21.4 \%)$ patients and bilateral therapeutic lateral lymph node dissection was performed in 31 (5.4\%) patients. In our center, total/ near-total thyroidectomy was routinely performed for PTC. CND included the removal of all fibroadipose tissue between the carotid sheath and trachea, from the hyoid bone inferiorly to the subclavian or innominate artery superiorly. After initial surgery, radioactive iodine therapy was recommended for the presence of extrathyroidal invasion, central LNM (CLNM), and lateral LNM (LLNM). All patients were advised to take levothyroxine for thyroid stimulating hormone suppression and to receive regular follow-up examination.

There was no permanent recurrent laryngeal nerve palsy or permanent hypoparathyroidism. Temporary hypoparathyroidism was found in $46(8.1 \%)$ patients. However, only $19(3.3 \%)$ patients had symptoms of hypocalcemia. Vocal cord palsy was found in 6 (1.1\%) patients. Postoperative hemorrhage developed in 14 (2.5\%) patients who received emergency surgery to control the bleeding.

All the pathological diagnoses of surgical specimens were performed carefully by three pathologists with over 10 years' experience at our institution. All cases were confirmed as PTC by using intraoperative frozen paraffin sections and postoperative paraffin sections. Single lobe diseases were found in $411(72.2 \%)$ patients with PTC, and bilateral in $158(27.8 \%)$ patients with PTC. Multifocality, considered as tumors $\geq 2$ foci, was found in one or both lobes, and the largest dimension was used for analysis in multifocal tumors. In our study, a $2-\mathrm{cm}$ tumor size cutoff value was used for analysis. Pathological tumor size $\leq 1.0 \mathrm{~cm}$ was found in $68.5 \%$ (390 of 569 cases) of patients, and $31.5 \%$ (179 of 569 cases) of patients were found with pathological tumor size $>1.0 \mathrm{~cm}$. The pathological examination revealed that $158(27.8 \%)$ cases were bilateral, 182 $(32.0 \%)$ were multifocal, 351 (61.6\%) were with CLNM, 122 (21.4\%) were with LLNM, 187 (32.8\%) had capsular invasion, 29 (5.1\%) had lymphovascular invasion, and 98 (17.2\%) had extrathyroidal invasion. In 351 patients with CLNM, the mean number of lymph nodes with metastasis was $3.57 \pm 2.93$ (range 1-15). All patients were classified to different stages according to the American Joint Committee on Cancer (AJCC) criteria [20, 21]: 416 (73.1\%) patients were in stage I, 8 (1.4\%) were in stage II, 111 (19.5\%) were in stage III, and $34(6.0 \%)$ were in stage IV. According to the American Thyroid Association (ATA) recurrence stratification criteria [22], there were 293 (51.5\%) patients with low recurrence stratification, $229(40.2 \%)$ with intermediate recurrence stratification, and $47(8.3 \%)$ with high recurrence stratification.

\section{Statistical Analysis}

Data were collected by using Microsoft Excel. The SPSS 20.0 package program was used to perform the statistical analysis. Data were presented as the means \pm SD. Univariate analyses using oneway ANOVA or Pearson's $\chi^{2}$ test was performed to estimate the relationships between HBsAg (or CLNM) and clinicopathological variables. Multivariate analysis was performed by using binary logistic regression. A $p$ value $<0.05$ was considered statistically significant.

\section{Results}

\section{Correlations between HBsAg and Clinicopathologic} Features of PTC

In this study, $14.8 \%$ (84 of 569 cases) of patients were HBsAg positive. The results of the comparative analysis 
Table 1. Analysis of the correlations between HBsAg and clinicopathologic characteristics of PTC

\begin{tabular}{|c|c|c|c|}
\hline & \multicolumn{2}{|l|}{ HBsAg } & \multirow{2}{*}{$\begin{array}{l}p \\
\text { value }\end{array}$} \\
\hline & negative & positive & \\
\hline Gender & & & 0.03 \\
\hline Male & $141(29.1)$ & $38(45.2)$ & \\
\hline Female & $344(70.9)$ & $46(54.8)$ & \\
\hline Age & & & 0.956 \\
\hline$<45$ years & $296(61.0)$ & $51(610)$ & \\
\hline$\geq 45$ years & $189(39.0)$ & $33(39.0)$ & \\
\hline Pathologic N stage & & & 0.039 \\
\hline pNo & $190(39.2)$ & $23(27.4)$ & \\
\hline $\mathrm{pN} 1$ & $295(60.8)$ & $61(72.6)$ & \\
\hline Bilaterality & & & 0.043 \\
\hline No & $358(73.8)$ & $53(63.1)$ & \\
\hline Yes & $127(25.2)$ & $31(36.9)$ & \\
\hline LLNM & & & 0.251 \\
\hline No & $385(79.4)$ & $62(73.8)$ & \\
\hline Yes & $100(20.6)$ & $22(26.2)$ & \\
\hline CLNM & & & 0.047 \\
\hline No & $194(40.0)$ & $24(28.6)$ & \\
\hline Yes & $291(60.0)$ & $60(71.4)$ & \\
\hline Pathologic tumor size & & & 0.512 \\
\hline$\leq 1.0 \mathrm{~cm}$ & $335(69.1)$ & $55(65.5)$ & \\
\hline$>1.0 \mathrm{~cm}$ & $150(30.9)$ & $29(35.5)$ & \\
\hline Multifocality & & & 0.295 \\
\hline No & $334(68.9)$ & $53(63.1)$ & \\
\hline Yes & $151(31.1)$ & $31(36.9)$ & \\
\hline Capsular invasion & & & 0.686 \\
\hline No & $324(66.8)$ & $58(69.0)$ & \\
\hline Yes & $161(33.2)$ & $26(31.0)$ & \\
\hline Lymphovascular invasion & & & 0.144 \\
\hline No & $463(95.5)$ & $77(91.7)$ & \\
\hline Yes & $22(4.5)$ & $7(8.3)$ & \\
\hline Extrathyroidal invasion & & & 0.868 \\
\hline No & $402(82.9)$ & $69(82.1)$ & \\
\hline Yes & $83(17.1)$ & $15(17.9)$ & \\
\hline
\end{tabular}

Data are presented as $n(\%)$. HBsAg, hepatitis B surface antigen; PTC, papillary thyroid carcinoma; $\mathrm{pN}$, pathologic N stage; LLNM, lateral lymph node metastasis; CLNM, central lymph node metastasis.

of the clinicopathological features of PTC in HBsAg-positive and HBsAg-negative patients are shown in Table 1. Univariate analysis presented that positive HBsAg was significantly correlated with tumor bilaterality $(p=0.043)$, more advanced pathological $\mathrm{N}$ stage $(p=0.039)$, and higher CLNM rate $(p=0.047)$. The prevalence rate of positive HBsAg in male patients ( 21.2 vs. $11.9 \%, p=0.03$ ) was higher than that in female patients. The incidence of bilateral tumors in $\mathrm{HBsAg}$-positive patients was 36.9\% (31 of 84 cases), which is significantly higher than that in HBsAg-negative patients, which was $25.2 \%$ ( 127 of 485 cases) $(p=0.043)$. The proportion of pathological N stage I(pN1) cases in PTC patients with positive HBsAg was also significantly higher than in those with negative HBsAg $(72.6$
Table 2. Univariate analysis of the correlations between CLNM and clinicopathologic characteristics of PTC

\begin{tabular}{|c|c|c|c|}
\hline & \multicolumn{2}{|l|}{ CLNM } & \multirow{2}{*}{$\begin{array}{l}p \\
\text { value }\end{array}$} \\
\hline & negative & positive & \\
\hline Gender & & & 0.032 \\
\hline Male & $57(26.1)$ & $122(34.8)$ & \\
\hline Female & $161(73.9)$ & $229(65.2)$ & \\
\hline Age, years & & & 0.000 \\
\hline$<45$ & $110(50.5)$ & $237(67.5)$ & \\
\hline$\geq 45$ & $108(49.5)$ & $114(32.5)$ & \\
\hline Pathologic tumor size & & & 0.000 \\
\hline$\leq 1.0 \mathrm{~cm}$ & $169(77.5)$ & $221(63.0)$ & \\
\hline$>1.0 \mathrm{~cm}$ & $49(22.5)$ & $130(37.0)$ & \\
\hline Bilaterality & & & 0.000 \\
\hline No & $177(81.2)$ & $234(66.7)$ & \\
\hline Yes & $41(18.8)$ & $117(33.3)$ & \\
\hline Multifocality & & & 0.000 \\
\hline No & $169(77.5)$ & $218(62.1)$ & \\
\hline Yes & $49(22.5)$ & $133(37.9)$ & \\
\hline Capsular invasion & & & 0.300 \\
\hline No & $152(69.7)$ & $230(65.5)$ & \\
\hline Yes & $66(30.3)$ & $121(34.5)$ & \\
\hline Lymphovascular invasion & & & 0.107 \\
\hline No & $211(96.8$ & $329(93.7)$ & \\
\hline Yes & $7(3.2)$ & $22(6.3)$ & \\
\hline Extrathyroidal invasion & & & 0.561 \\
\hline No & $183(83.9)$ & $288(82.1)$ & \\
\hline Yes & $35(16.1)$ & $63(17.9)$ & \\
\hline LLNM & & & 0.000 \\
\hline No & $207(99.5$ & $240(68.4)$ & \\
\hline Yes & $11(0.5)$ & $111(31.6)$ & \\
\hline HBsAg & & & 0.047 \\
\hline Negative & $194(40.0)$ & $291(60.0)$ & \\
\hline Positive & $24(28.6)$ & $60(71.4)$ & \\
\hline
\end{tabular}

Data are presented as $n(\%)$. CLNM, central lymph node metastasis; PTC, papillary thyroid carcinoma; LLNM, lateral lymph node metastasis; HBsAg, hepatitis B surface antigen.

vs. $60.8 \%, p=0.039)$. In addition, the rate of CLNM in PTC patients with positive HBsAg was significantly higher than in those with negative HBsAg (71.4 vs. $60.0 \%, p=$ $0.047)$. No statistically significant differences were found in $\operatorname{LLNM}(p=0.251)$, multifocality $(p=0.295)$, tumor size $(p=0.512)$, vascular invasion $(p=0.144)$, capsular invasion $(p=0.686)$, or extrathyroidal invasion $(p=0.868)$.

\section{Correlations between CLNM and Clinicopathologic} Features of PTC

In our study, $61.7 \%$ (351 of 569 cases) of patients with PTC had CLNM. Univariate analysis presented that CLNM of patients with PTC was significantly correlated with gender $(p=0.032)$, age $(p=0.000)$, tumor size $(p=$ $0.000)$, bilaterality $(p=0.000)$, multifocality $(p=0.000)$, $\operatorname{LLNM}(p=0.000)$, and HBsAg $(p=0.047)$. Obviously, the 
Table 3. Multivariate analysis of the correlations between CLNM and clinicopathologic characteristics of PTC

\begin{tabular}{|c|c|c|c|c|c|c|c|c|}
\hline & \multirow[t]{2}{*}{ B } & \multirow[t]{2}{*}{ SE } & \multirow[t]{2}{*}{ Wals } & \multirow[t]{2}{*}{$\mathrm{df}$} & \multirow[t]{2}{*}{ Sig. } & \multirow[t]{2}{*}{$\operatorname{Exp}(B)$} & \multicolumn{2}{|c|}{$95 \% \mathrm{CI}$} \\
\hline & & & & & & & lower & upper \\
\hline Gender & -0.2 .99 & 0.208 & 2.059 & 1 & 0.151 & 0.742 & 0.493 & 1.116 \\
\hline Tumor size & 0.396 & 0.188 & 4.425 & 1 & 0.035 & 1.486 & 1.027 & 2.149 \\
\hline Bilaterality & 0.271 & 0.456 & 0.353 & 1 & 0.552 & 1.311 & 0.537 & 3.203 \\
\hline Age & -0.679 & 0.191 & 12.618 & 1 & 0.000 & 0.507 & 0.349 & 0.738 \\
\hline Multifocality & 0.416 & 0.427 & 0.948 & 1 & 0.330 & 1.515 & 0.656 & 3.498 \\
\hline Lateral LN metastasis & 1.933 & 0.337 & 32.890 & 1 & 0.000 & 6.909 & 3.569 & 13.375 \\
\hline HBsAg & 0.590 & 0.284 & 4.315 & 1 & 0.038 & 1.804 & 1.034 & 3.149 \\
\hline Constant & -0.220 & 0.676 & 0.106 & 1 & 0.745 & 0.803 & & \\
\hline
\end{tabular}

CLNM, central lymph node metastasis; PTC, papillary thyroid carcinoma; LLNM, lateral lymph node metastasis; HBsAg, hepatitis B surface antigen.

incidence of CLNM in male patients (122 of 179 cases, $68.2 \%)$ was higher than that in female patients ( 229 of 390 cases, $58.7 \%)(p=0.032)$. In PTC patients aged $<45$ years, $68.3 \%$ (237 of 347 ) of patients had CLNM, while only $51.4 \%$ ( 114 of 222 ) of patients aged $\geq 45$ years had CLNM. The statistical difference between these two groups was significant $(p=0.000)$. The probability of CLNM was higher in patients with pathologic tumor size $>1 \mathrm{~cm}(130$ of 179 cases, $72.6 \%$ ) compared with pathologic tumor size $\leq 1 \mathrm{~cm}(221$ of 390 cases, $56.7 \%)(p=0.000)$. The incidence of CLNM was $74.1 \%$ (117 of 158 cases) in patients with bilateral tumors, while only $56.9 \%$ (234 of 411 cases) of patients with unilateral tumor had CLNM. There was a statistically significant difference between these two groups ( $p=0.000$ ). Also, $73.1 \%$ (133 of 182 cases) of patients with multifocal tumor were detected to have CLNM, which is a higher incidence than in patients with unifocal tumor ( 218 of 387 cases, $56.3 \%)(p=0.000)$. Interestingly, the incidence of CLNM in patients with positive HBsAg was $71.4 \%$ (60 of 84 cases), which is higher than in patients with negative HBsAg $60.0 \%$ (291 of 485 cases) ( $p=$ 0.047 ). No statistically significant correlations were observed between CLNM and other characteristics such as capsular invasion $(p=0.300)$, extrathyroidal invasion $(p=0.561)$, and lymphovascular invasion $(p=0.107)(\mathrm{Ta}-$ ble 2). Multivariate analysis revealed that tumor size $>1$ $\mathrm{cm}(p=0.035)$, age $<45$ years $(p=0.000), \operatorname{LLNM}(p=$ $0.000)$, and positive HBsAg $(p=0.038)$ were independent predictors of CLNM (Table 3 ).

\section{Discussion}

In accordance with the guidelines of the ATA [23], total or near-total thyroidectomy is the standard surgical treatment for PTC with tumor size $>1 \mathrm{~cm}$ unless there are contraindications. There was consensus about the need to routinely perform therapeutic CND and therapeutic lateral lymph node dissection in PTC patients. Although it was controversial about whether routine prophylactic CND should be performed in clinical $\mathrm{N}$ stage $0(\mathrm{cN} 0)$ PTC patients, Chinese guidelines for thyroid nodule disease and differentiated thyroid carcinoma recommend CND for patients with PTC (Grade A) [24]. In our study, bilateral CND in addition to total/near-total thyroidectomy was performed in all patients. It has been reported that LNM was associated with increased recurrence and influenced survival in PTC patients $[4,25]$. It has also been reported that the incidence of LNM in PTC patients was $33.3 \%$, which was higher than patients with PTMC [6]. Our previous study reported that the rate of CLNM in patients with PTC was 63.2\% [7]. The Chinese second national survey of HBV sero-epidemiology showed that the general prevalence rate of HBsAg was 7.18\% in 2006 [26]. Kamiza et al. [15] reported that HBV infection was associated with an increased risk of developing thyroid gland cancer. However, whether HBV infection affects the progression of LNM in PTC has not been reported in the literature. Therefore, we analyzed the relationship between HBsAg and LNM in this study.

A review demonstrated that the prevalence rate of serum HBsAg was greater than $8 \%$ in Southern provinces of China [27]. Our study was carried out in Guangdong province (one of Southern provinces of China), and the incidence of positive HBsAg among PTC patients in this study was $14.8 \%$ ( 84 of 569 cases), which is higher than that reported in the Chinese second national survey (7.18\%) [26]. The prevalence rate of positive HBsAg in male patients ( 21.2 vs. $11.9 \%, p=0.03$ ) was higher than the rate in female patients. Some studies [28-30] also reported that the prevalence rate of positive HBsAg in male patients is higher. The incidence of bilateral tumors in HBsAg-positive patients was $36.9 \%$ ( 31 of 84 cases), higher than that in HBsAg-negative patients (25.2\%, 127 of 485 
cases) ( $p=0.043)$. It reminds us that we should pay more attention to bilateral tumors when patients are positive for HBsAg. No statistically significant correlations were observed between HBsAg and other characteristics such as LLNM $(p=0.251)$, tumor size $(p=0.512)$, multifocality $(p=0.295)$, capsular invasion $(p=0.686)$, lymphovascular invasion ( $p=0.144)$, or extrathyroidal invasion $(p=0.868)$.

In our study, CLNM were found in 61.7\% (351 of 569 cases) of patients with PTC. The incidence of CLNM in PTC patients with positive HBsAg was significantly higher than that of patients with negative HBsAg (71.4 vs. $60.0 \%, p=0.047$ ). And the proportion of patients with pathological N stage I ( $\mathrm{pN} 1$ ) in PTC patients with positive HBsAg was also significantly higher (72.6 vs. $60.8 \%, p=$ 0.039). Thus, it indicated that positive HBsAg was significantly correlated with LNM in PTC patients, especially CLNM. Multivariate logistic regression analysis indicated that positive $\mathrm{HBsAg}(p=0.038)$ was an independent predictor of CLNM in PTC patients. Therefore, we should carefully perform complete CND when $\mathrm{HBsAg}$-positive patients have PTC. However, another study concerning breast cancer patients with HBV infection showed that the metastatic axillary lymph node ratio in breast cancer patients with positive HBsAg was significantly lower than that in patients with negative HBsAg ( 33.1 vs. $42.8 \%, p=$ 0.04) [31]. This conclusion was obviously the opposite to that in our study, which means that different types of cancer may have different outcomes.

A variety of viruses have played an important role in thyroid cancer. Etemadi et al. [32] reported that human parvovirus B19 infection may play an important role in tumorigenesis and thyroid cancer development via the inflammatory mechanisms. Homayouni et al. [33] demonstrated that Epstein-Barr virus may play a role in PTC, especially in younger patients. Antonelli et al. [34] reported that patients with HCV infection had a high prevalence of thyroid papillary cancer and were overall in a state of thyroid autoimmunity. To date, the role of HBV in thyroid cancer has not been reported. Our study demonstrated that positive HBsAg was a risk factor of LNM and bilateral tumors in PTC patients and an independent predictor of CLNM in PTC patients.

Vascular endothelial growth factor (VEGF) can inhibit the function of antigen-presenting cells which play an important role in the innate immune response to tumors [35]. Previous studies have found that an increased level of VEGF along with VEGF-C and their receptors VEGFR-2 and VEGFR-3 are related with LNM of PTC [36, 37]. Muhanna et al. [38] reported that VEGF level was significantly increased in mice with hepatic fibrosis due to liver function damage. HBV infection can lead to chronic liver injury and then cause hepatic fibrosis. These can all damage liver function. Serum VEGF level may increase in patients with HBV infection due to liver func- tion damage. With the increase of VEGF level, the risk of LNM will increase in patients with PTC.

There are several limitations to this study. Firstly, it was a retrospective study performed from a single center, and there might have been a selection bias. Secondly, the follow-up period was relatively short in our study, and we could not evaluate the long-term prognosis of PTC patients with positive HBsAg. Therefore, a large-sample multicenter prospective study may need to be performed to confirm these findings.

\section{Conclusion}

The incidence of CLNM in PTC patients with positive HBsAg was significantly higher than that in patients with negative HBsAg. Positive HBsAg was correlated with LNM and bilateral tumors. In addition to gender, age $<45$ years, tumor size $>2 \mathrm{~cm}$, and LLNM, positive HBsAg was also an independent predictor of CLNM in PTC patients.

\section{Statement of Ethics}

This study was approved by the ethics committee of Guangdong Provincial People's Hospital. Informed consent was obtained from all individual participants included in the study. The authors have no ethical conflicts to disclose.

\section{Disclosure Statement}

The authors have no conflicts of interest to declare.

\section{Funding Sources}

The authors have no funding sources to disclose.

\section{Author Contributions}

J.Y. analyzed and interpreted the patient data regarding the clinicopathologic features of conventional PTC and HBsAg. X.C. performed the data collection. Z.Z. was a major contributor in writing the manuscript. All authors read and approved the final manuscript.

References

1 Chen W, Zheng R, Baade PD, Zhang S, Zeng $\mathrm{H}$, Bray $\mathrm{F}$, et al. Cancer statistics in China, 2015. CA Cancer J Clin. 2016 Mar-Apr;66(2): $115-32$.

2 Siegel RL, Miller KD, Jemal A. Cancer Statistics, 2017. CA Cancer J Clin. 2017 Jan;67(1): 7-30.

3 DeSantis CE, Lin CC, Mariotto AB, Siegel RL, Stein KD, Kramer JL, et al. Cancer treatment and survivorship statistics, 2014. CA Cancer J Clin. 2014 Jul-Aug;64(4):252-71. 
4 Lundgren CI, Hall P, Dickman PW, Zedenius J. Clinically significant prognostic factors for differentiated thyroid carcinoma: a population-based, nested case-control study. Cancer. 2006 Feb;106(3):524-31.

5 Hedinger C, Williams ED, Sobin LH. The WHO histological classification of thyroid tumors: a commentary on the second edition. Cancer. 1989 Mar;63(5):908-11.

6 Karatzas T, Vasileiadis I, Kapetanakis S, Karakostas E, Chrousos G, Kouraklis G. Risk factors contributing to the difference in prognosis for papillary versus micropapillary thyroid carcinoma. Am J Surg. 2013 Oct;206(4):58693.

7 Yuan J, Zhao G, Du J, Chen X, Lin X, Chen Z, et al. To identify predictors of central lymph node metastasis in patients with clinically node-negative conventional papillary thyroid carcinoma. Int J Endocrinol. 2016;2016: 6109218.

8 Trépo C, Chan HL, Lok A. Hepatitis B virus infection. Lancet. 2014 Dec;384(9959):205363.

9 Liaw YF, Chu CM. Hepatitis B virus infection. Lancet. 2009 Feb;373(9663):582-92.

10 Chen GF, Wang C, Lau G. Treatment of chronic hepatitis B infection-2017. Liver Int. 2017 Jan;37 Suppl 1:59-66.

11 Schweitzer A, Horn J, Mikolajczyk RT, Krause G, Ott JJ. Estimations of worldwide prevalence of chronic hepatitis B virus infection: a systematic review of data published between 1965 and 2013. Lancet. 2015 Oct;386(10003): 1546-55.

12 Te HS, Jensen DM. Epidemiology of hepatitis $B$ and $C$ viruses: a global overview [vii.]. Clin Liver Dis. 2010 Feb;14(1):1-21.

13 Sundaram V, Kowdley K. Management of chronic hepatitis B infection. BMJ. 2015 Oct; 351:h4263.

14 Zampino R, Sagnelli C, Boemio A, Sagnelli E, Coppola N. Treatment of chronic HBV infection in developing countries. Ann Hepatol. 2016 Nov-Dec;15(6):816-23.

15 Kamiza AB, Su FH, Wang WC, Sung FC, Chang SN, Yeh CC. Chronic hepatitis infection is associated with extrahepatic cancer development: a nationwide population-based study in Taiwan. BMC Cancer. 2016 Nov; 16(1):861.

16 Wei XL, Luo HY, Li CF, Jin Y, Zeng ZL, Ju $\mathrm{HQ}$, et al. Hepatitis B virus infection is associated with younger median age at diagnosis and death in cancers. Int J Cancer. 2017 Jul 141(1):152-9.
17 Patel BB, Lipka S, Shen H, Davis-Yadley AH, Viswanathan P. Establishing the link between hepatitis B virus infection and colorectal adenoma. J Gastrointest Oncol. 2015 Oct;6(5): 492-7.

18 Wei XL, Qiu MZ, Chen WW, Jin Y, Ren C, Wang F, et al. The status of HBV infection influences metastatic pattern and survival in Chinese patients with pancreatic cancer. J Transl Med. 2013 Oct;11(1):249.

19 Wei XL, Qiu MZ, Jin Y, Huang YX, Wang RY, Chen WW, et al. Hepatitis B virus infection is associated with gastric cancer in China: an endemic area of both diseases. Br J Cancer. 2015 Mar;112(7):1283-90.

20 Wada N, Nakayama H, Suganuma N, Masudo Y, Rino Y, Masuda M, et al. Prognostic value of the sixth edition AJCC/UICC TNM classification for differentiated thyroid carcinoma with extrathyroid extension. J Clin Endocrinol Metab. 2007 Jan;92(1):215-8.

21 Stratmann M, Sekulla C, Dralle H, Brauckhoff M. [Current TNM system of the UICC/AJCC : the prognostic significance for differentiated thyroid carcinoma]. Chirurg. 2012 Jul;83(7): 646-51.

22 Pitoia F, Bueno F, Urciuoli C, Abelleira E, Cross G, Tuttle RM. Outcomes of patients with differentiated thyroid cancer risk-stratified according to the American thyroid association and Latin American thyroid society risk of recurrence classification systems. Thyroid. 2013 Nov;23(11):1401-7.

23 Haugen BR, Alexander EK, Bible KC, Doherty GM, Mandel SJ, Nikiforov YE, et al. 2015 American Thyroid Association Management Guidelines for Adult Patients with Thyroid Nodules and Differentiated Thyroid Cancer: The American Thyroid Association Guidelines Task Force on Thyroid Nodules and Differentiated Thyroid Cancer. Thyroid. 2016 Jan;26(1):1-133.

24 Teng WP, Gao M, Huang G. Thyroid nodules and differentiated thyroid cancer treatment guidelines. Chin J Clin Oncol. 2012;39(17): 1249-72.

25 Liu FH, Kuo SF, Hsueh C, Chao TC, Lin JD. Postoperative recurrence of papillary thyroid carcinoma with lymph node metastasis. J Surg Oncol. 2015 Aug;112(2):149-54.

$26 \mathrm{Yu} \mathrm{R}$, Fan R, Hou J. Chronic hepatitis B virus infection: epidemiology, prevention, and treatment in China. Front Med. 2014 Jun; $8(2): 135-44$

27 Lu FM, Li T, Liu S, Zhuang H. Epidemiology and prevention of hepatitis $\mathrm{B}$ virus infection in China. J Viral Hepat. 2010 Mar;17 Suppl 1: 4-9.
28 Chan SL, Wong VW, Qin S, Chan HL. Infection and Cancer: The Case of Hepatitis B. J Clin Oncol. 2016 Jan;34(1):83-90.

29 Sundquist K, Sundquist J, Ji J. Risk of hepatocellular carcinoma and cancers at other sites among patients diagnosed with chronic hepatitis B virus infection in Sweden. J Med Virol. 2014 Jan;86(1):18-22.

30 Deutsch M, Dourakis S, Manesis EK, Gioustozi A, Hess G, Horsch A, et al. Thyroid abnormalities in chronic viral hepatitis and their relationship to interferon alfa therapy. Hepatology. 1997 Jul;26(1):206-10.

31 Wu H, Zhao C, Adhikari VP, Lu L, Huang J, Wei Y, et al. The prevalence and clinicopathological features of breast cancer patients with hepatitis B virus infection in China. Oncotarget. 2017 Mar;8(11):18185-90.

32 Etemadi A, Mostafaei S, Yari K, Ghasemi A, Minaei Chenar H, Moghoofei M. Detection and a possible link between parvovirus B19 and thyroid cancer. Tumour Biol. 2017 Jun; 39(6): 1010428317703634

33 Homayouni M, Mohammad Arabzadeh SA, Nili F, Razi F, Amoli MM. Evaluation of the presence of Epstein-Barr virus (EBV) in Iranian patients with thyroid papillary carcinoma. Pathol Res Pract. 2017 Jul;213(7):854-6.

34 Antonelli A, Ferri C, Fallahi P, Pampana A, Ferrari SM, Barani L, et al. Thyroid cancer in HCV-related chronic hepatitis patients: a case-control study. Thyroid. 2007 May; 17(5): 447-51.

35 Dvorak HF, Nagy JA, Feng D, Brown LF, Dvorak AM. Vascular permeability factor/ vascular endothelial growth factor and the significance of microvascular hyperpermeability in angiogenesis. Curr Top Microbiol Immunol. 1999;237:97-132.

36 Nakamura Y, Yasuoka H, Zuo H, Takamura Y, Miyauchi A, Nakamura M, et al. Nitric oxide in papillary thyroid carcinoma: induction of vascular endothelial growth factor D and correlation with lymph node metastasis. J Clin Endocrinol Metab. 2006 Apr;91(4): 1582-5.

37 Yu XM, Lo CY, Chan WF, Lam KY, Leung P, Luk JM. Increased expression of vascular endothelial growth factor $\mathrm{C}$ in papillary thyroid carcinoma correlates with cervical lymph node metastases. Clin Cancer Res. 2005 Nov; 11(22):8063-9.

38 Muhanna N, Amer J, Salhab A, Sichel JY, Safadi R. The immune interplay between thyroid papillary carcinoma and hepatic fibrosis. PLoS One. 2015 Jul;10(7):e0132463. 